\title{
Hedgehog-Patched pathway aberrations in a malignant triton tumor case study
}

\author{
MILJENKO BURA $^{1}$, VESNA MUSANI ${ }^{2}$, MAJA CRETNIK $^{2}$ IVA BOTICA $^{1}$ and SONJA LEVANAT ${ }^{2}$ \\ ${ }^{1}$ Department of Otorhinolaryngology and Head and Neck Surgery, Clinical Hospital Center 'Rebro', Kispaticeva 12; \\ ${ }^{2}$ Division of Molecular Medicine, Rudjer Boskovic Institute, Bijenicka 54, 10000 Zagreb, Croatia
}

Received January 25, 2008; Accepted April 16, 2008

DOI: 10.3892/or_00000013

\begin{abstract}
Transition from malignant schwannoma to malignant triton tumor is analyzed in a case report on a patient with recurring cancers and suspected familial predisposition. It is hypothesized that rhabdomyoblastic differentiation, which distinguishes triton from schwannoma, might be attributable to Hedgehog-Patched pathway malfunctioning. Loss of one Patched gene allele was found in the tissue of advanced triton, but the retained allele had no exon or promoter mutations. Protein levels at early cancer stages indicated possible Patched response to the pathway activation in the first occurrence of triton tumor. Later, in the recurring triton, Patched expression was several times lower than in the control tissue, suggesting that haploinsufficiency was aided by silencing of the remaining allele, although its promoter was not hypermethylated. These findings may justify further investigation of the HedgehogPatched pathway role in triton malignancies, especially because of the recent research on the therapeutical potential of the pathway.
\end{abstract}

\section{Introduction}

Malignant triton tumor (MTT) is a very rare variant of malignant schwannoma with rhabdomyoblastic differentiation, which carries a poor prognosis despite wide excision, chemotherapy, and radiotherapy. This aggressive tumor, arising in the peripheral nerve sheath, is comprised of both malignant schwannoma cells and malignant rhabdomyoblasts. Less than one hundred cases were reported in the twentieth century (1), after the first case described in 1932 (2). MTT predominantly affects young patients, and is associated with neurofibromatosis type 1 (NF1) in $>50 \%$ cases ( 3 ). In addition, less than one third of triton malignancies are located in the head and neck

Correspondence to: Dr Sonja Levanat, Division of Molecular Medicine, Rudjer Boskovic Institute, Bijenicka 54, 10000 Zagreb, Croatia

E-mail: levanat@irb.hr

Key words: triton tumor, Hedgehog-Patched pathway, PTCH1, schwannoma region (4), so that the intracranial tumor of an elderly woman with no symptoms of NF1, which is reported here, belongs to a very small subset of MTT cases.

Schwann cells derive from the neural crest via Schwann cell precursor, in myelating or non-myelating form (5). Schwannomas are usually slowly growing tumors, derived from Schwann cells, and surrounding the peripheral, cranial or autonomic nerves. They occur at a wide variety of sites, frequently within the head and neck region, and account for about $8 \%$ of intracranial and $29 \%$ of spinal tumors (6).

Malignant schwannoma is a rare aggressive schwannoma variant, with prognosis not much better than MTT. About $30 \%$ of malignant schwannoma cases occur sporadically of unknown etiology, as the sole morbid finding. The remaining $70 \%$ are associated with NF1; their biological behaviour is more aggressive than that of simple malignant schwannomas; their course is rapid and the prognosis is dismal. Local recurrences are frequent, and distant metastases, preferentially situated in the lung and brain, are present in about $50 \%$ of the cases.

We report on a family with two members affected by malignant schwannoma; in one patient schwannoma developed into MTT, whereas the fate of the other patient is not known. Our study was primarily directed towards genetic aspects which could be relevant for development and progression of the triton malignancy, focusing on the Hedgehog-Patched signaling pathway. The pathway was selected because of its known role in Schwann cells development, and because of its implication in rhabdomyosarcoma.

The Hedgehog-Patched signaling pathway is crucial for the morphogenesis of nervous system. It is involved in neural cell precursor proliferation and regulates neural stem cells in various stages of development. Sporadic brain tumorigenesis may be attributable to inappropriate activation or inactivation of the pathway $(7,8)$. The Hedgehog $(\mathrm{Hh})$ family in mammals consists of three members: Shh (Sonic Hedgehog), Dhh (Desert Hedgehog) and Ihh (Indian Hedgehog). Dhh is secreted from Schwann cells and regulates the formation of surrounding tissues (5), but in general Shh is more frequently found to be involved in normal and especially in aberrant activation of the pathway. When any Hh ligand binds to the transmembrane receptor protein Patched (Ptc, in humans Ptch1), the co-receptor Smoothened (Smo) is relieved from repression by Ptc, which triggers signal transduction cascade within the cell. At the downstream end of the pathway, the 


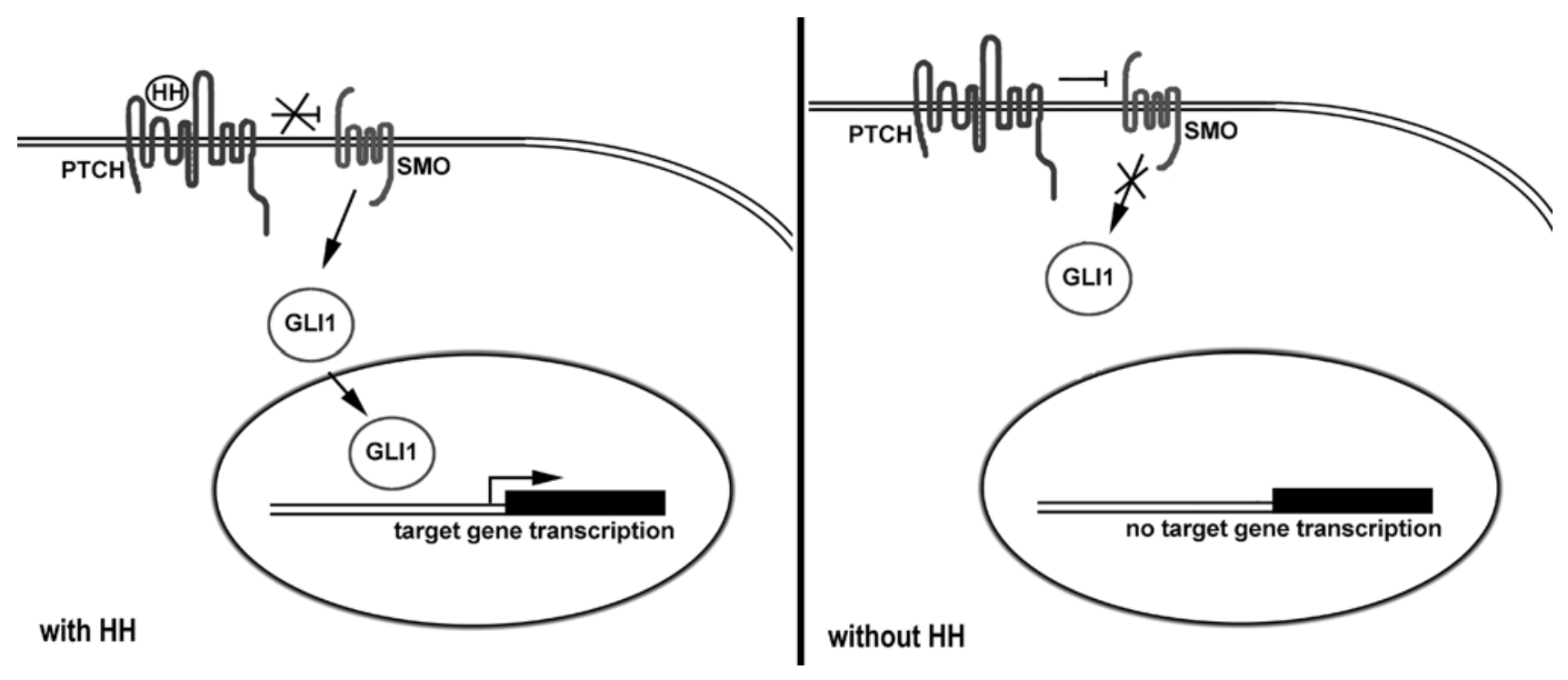

Figure 1. Shematic presentation of the main members of the Hh-Ptc signaling pathway.

active form of Gli transcription factor is translocated to the nucleus, and expression of the pathway target genes is stimulated (Fig. 1), which leads to cell proliferation. Gli1, Gli2 and Gli3 proteins exibit different functions in different tissues, and are generally involved in patterning of the nervous system and brain development. In particular, the active form of Gli1 binds to PTC promoter and stimulates its expression, so that new Ptc protein may limit and eventually terminate the pathway activity. When this autoregulatory mechanism is impaired, frequently by genetic or epigenetic alterations of PTC, malformations or cancer may develop, which has been particularly convincingly demonstrated on mouse models of rhabdomyosarcoma (9).

In addition to the analysis of the pathway genes PTCH1, SHH, SMO and GLI1, we also examined p16 as a potential regulator of cell cycle progression and proliferation in Schwann cells (10) and NF1 gene for its potential role in schwannoma development (11). PCNA expression was measured as a general indicator of cell proliferation.

\section{Materials and methods}

Subjects and samples. Blood and tissue samples from three consecutive tumors (in liquid nitrogen and in paraffin block) of one patient $(\mathrm{SCH})$ were analyzed. Her sister (S3), diagnosed with malignant schwannoma, was not available for analysis. Blood samples were obtained from four unaffected relatives S1, S2, S4, S5 (family tree in Fig. 2).

Patient's history. Female patient (SCH) had first symptoms (swelling and pain in the inner corner of the right eye) in 1999, at the age of 68. After clinical exams (echography, CT) and tumor removal, pathohistological diagnosis (PHD) of malignant schwannoma was given. Several months later, in the spring of 2000, a new tumor of the right orbit was detected, exenteration of orbit was performed, and PHD was triton malignum. The patient was irradiated, but at the end of 2000 lymphadenopathia was detected, and biopsy of lymph node in submandibular area showed malignant cells; radical neck dissection was done, and PHD was determined as metastaticum lymphonodorum. FACS analysis showed aneuploidy and high mitotic index, and patient's neck was again irradiated. In mid 2001, the patient had overgrowth of parotid gland, complete parotidectomy was done, and PHD was triton malignum rec. At the beginning of 2002, new tumor located preauriculary was resected (again triton malignum rec). In the following months the patient had serious loss of weight, bone scintigraphy showed metastatic process, and she died in November, 2002. Table I lists tumor samples that were available for analysis and the tests we performed.

DNA extraction and PCR. DNA samples were extracted from blood leukocytes and from frozen tumor or paraffin tissues by standard methods. DNA samples were typed for ten short tandem repeat polymorphisms, D9S196, WI-19346, GDB:583921, PTCH intra, ex 1 UTR, D9S287, D9S180, D9S176, D9S1783 and D9S127, spanning chromosome region 9q22.3-31 (12). Intragenic $\mathrm{PTCH}$ is located in the first intron of PTCH1 between exons 1 and 2 (13). Polymorphic marker ex 1 UTR is located upstream of exon 1 (14). All other primer sequences for polymorphic markers were taken from online NCBI database (www.ncbi.nlm.nih.gov).

PCR reaction was performed in $25 \mu 1$ reaction mixture using Multiplex PCR kit (Qiagen) containing 200-300 ng of template DNA and 8 to $20 \mathrm{pmol} / \mathrm{l}$ of each primer pair. PCR system (GeneAmp PCR system 2400, Applied Biosystems) was set for 19-25 cycles (15).

For LOH analysis fluorescent forward primers were used. Multiplex PCR products $(1 \mu \mathrm{l})$ was mixed with $12.5 \mu \mathrm{l}$ of HiDi formamide (Applied Biosystems) and 0.5 $\mu \mathrm{l}$ GeneScan 500 LIZ size standard (Applied Biosystems), and loaded on ABI PRISM 310 automatic sequencer (Applied Biosystems). The analysis was made using Gene Mapper v3.0.

For three markers in 9p21 (IFNA, D9S126 and D9S104), PCR reaction was performed in $25 \mu 1$ reaction mixture 

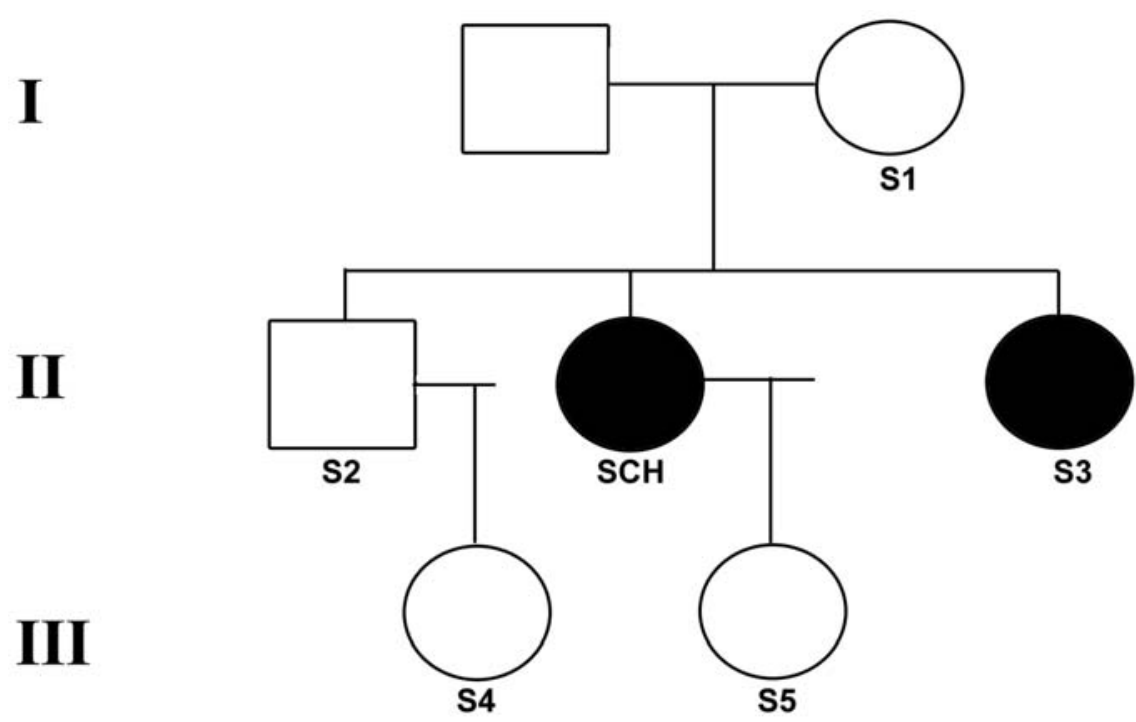

\begin{tabular}{|l|c|c|c|c|c|c|}
\hline $\begin{array}{l}\text { polymorphic } \\
\text { marker }\end{array}$ & S2 & S4 & SCH & S5 & S1 & S3 \\
\hline D9S196 & 1,2 & 2,2 & 2,2 & 2,2 & 1,2 & - \\
\hline WI-19346 & 1,2 & 1,3 & 1,3 & 3,3 & 1,1 & - \\
\hline GDB:583921 & 1,1 & 1,1 & 1,2 & 1,2 & 1,1 & - \\
\hline PTCH intra & 1,1 & 1,2 & 1,2 & 2,2 & 1,2 & - \\
\hline ex 1 UTR & 2,2 & 1,2 & 1,2 & 1,1 & 1,2 & - \\
\hline D9S287 & 1,2 & 2,3 & 2,5 & 2,5 & 2,3 & - \\
\hline D9S180 & 1,5 & 1,1 & 1,5 & 5,6 & 1,6 & - \\
\hline D9S176 & 1,2 & 2,2 & 2,4 & 4,4 & 2,2 & - \\
\hline D9S1783 & 1,3 & 1,3 & 2,3 & 2,2 & 1,3 & - \\
\hline D9S127 & 4,4 & 2,4 & 4,4 & 1,4 & 1,4 & - \\
\hline
\end{tabular}

Figure 2. Linkage analysis was performed with ten polymorphic markers for the 9q22.3-31 region. SCH is the patient with MTT, S3 is her sister diagnosed with malignant schwannoma, other relatives are unaffected.

Table I. Tumor samples and tests performed.

\begin{tabular}{lll}
\hline Sample label and date & \multicolumn{1}{c}{ Tumor diagnosis } & \multicolumn{1}{c}{ Tests performed } \\
\hline PHD1, September 1999 & Malignant schwannoma & Immunohistochemistry \\
PHD2, March 2000 & Triton malignum & Immunohistochemistry \\
PHD3, January 2002 & Triton malignum rec & LOH analysis, PTCH1 exon sequencing and promoter \\
& & methylation, RFLP, RT-PCR expression analysis \\
\hline
\end{tabular}

containing $100 \mathrm{ng}$ of template DNA, $200 \mu \mathrm{mol} / 1$ deoxynucleoside triphosphate (dNTP), $1.5 \mathrm{mmol} / \mathrm{l} \mathrm{MgCl}_{2}, 10 \mathrm{pmol} / \mathrm{l}$ each primer, 1.25 U Taq polymerase (AmpliTag Gold ${ }^{\circledR}$ DNA polymerase, Applied Biosystems) in $10 \mathrm{mmol} / \mathrm{l}$ Tris- $\mathrm{HCl}$ buffer. PCR system (GeneAmp PCR system 2400, Applied Biosystems) was set for 25-35 cycles, with the parameters published previously (16). LOH analysis was performed on 8-12\% polyacrylamide gel, as described previously (17). LOH analysis of NF1 locus was performed by RFLP (18).

Mutation analysis. All 23 exons of PTCH1 gene (www.ncbi. nlm.nih.gov) were PCR amplified, subjected to additional 10 min $95^{\circ} \mathrm{C}$ denaturating step, followed by 30 -min reannealing, and then loaded on the dHPLC (denaturing high-performance liquid chromatography) WAVE DNA Fragment Analysis System (Transgenomic, Omaha, NE, USA). Twenty-three exons of PTCH1 and 3 exons of p16 gene were sequenced in both directions using the Big Dye Terminator v.1.1 Cycle Sequencing kit and analyzed on ABI PRISM 310 Genetic Analyser using DNA sequencing Analysis Software version 5.1. (Applied Biosystems).

Real-time PCR (RT-PCR). Total cellular RNA was isolated from tissue samples of triton (PHD3) and healthy neural tissue (Nervus auricularis magnus, nerve from plexus cervicalis, the closest in origin to normal tissue). 
Table II. Typing with polymorphic markers for $9 \mathrm{p} 21$ and 9q22-31.

\begin{tabular}{lcc}
\hline Polymorphic marker & SCH blood & SCH tumor \\
\hline IFNA & 1,2 & 1,2 \\
D9S126 & 1,1 & 1,1 \\
D9S104 & 1,2 & 1,2 \\
D9S196 & 2,2 & $2, \mathrm{LOH}$ \\
WI-19346 & 1,3 & $1, \mathrm{LOH}$ \\
GDB:583921 & 1,2 & $1, \mathrm{LOH}$ \\
PTCH intra & 1,2 & $1, \mathrm{LOH}$ \\
ex 1 UTR & 1,2 & LOH,2 \\
D9S287 & 2,5 & $2, \mathrm{LOH}$ \\
D9S180 & 1,5 & $1, \mathrm{LOH}$ \\
D9S176 & 2,4 & $2, \mathrm{LOH}$ \\
D9S1783 & 2,3 & LOH,3 \\
D9S127 & 4,4 & $4, \mathrm{LOH}$ \\
\hline
\end{tabular}

${ }^{a}$ Allelic fragments are numbered from the smallest to the highest.

The isolation was performed in two steps: first, tissue was homogenized in TRIzol reagent (Invitrogen), RNA was extracted, washed with $75 \%$ ethanol and dissolved in water and, as a second step, run through High Pure RNA Isolation Kit columns (Roche), and finally dissolved in $30 \mu 1$ elution buffer. Complementary DNA (cDNA) was synthesized from each of the samples starting from $1 \mu \mathrm{g}$ of total RNA, using TaqMan reverse transcription kit (Applied Biosystems).

Real-time PCR analysis was performed on Chromo4 Real-time PCR system (Bio-Rad), in total volume of $10 \mu 1$, using iQ SYBR Green supermix (Bio-Rad). Expression of 5 different genes was analyzed, with Arp (acidic ribosomal protein) as a reference housekeeping gene (19). All samples were done in triplicates, and blanks were included. Primers used for real-time PCR amplification were published previously (20). Real-time PCR program was: $95^{\circ} \mathrm{C}$ for $3 \mathrm{~min}$, and 45 cycles of $95^{\circ} \mathrm{C}$ for $15 \mathrm{sec}$ and $61^{\circ} \mathrm{C}$ for $1 \mathrm{~min}$, followed by plate read step. Product specificity was determined by melting curve analysis and on a $2 \%$ agarose gel stained with ethidium bromide. Data were analyzed using the $2^{-\Delta \Delta C t}$ (relative quantitation) method, after ensuring that all primer sets amplified their respective sequence targets with similar efficiencies.

Immunohistochemistry. Tumor paraffin sections were deparaffinated, then incubated at $95-99^{\circ} \mathrm{C}$ for $40 \mathrm{~min}$ in Epitope Retrieval Solution (Dako) and cooled to room temperature. Endogenous peroxidase was blocked with methanol-hydrogen peroxide solution (10 ml 30\% $\mathrm{H}_{2} \mathrm{O}_{2}$ in $90 \mathrm{ml}$ methanol) for $10 \mathrm{~min}$, and Protein-block Serum-Free (Dako) was applied for $10 \mathrm{~min}$. Primary rabbit anti-Ptch 1 antibody (dilution 1:500) (gift from Allen Bale), Gli1 (ab7523, abcam), or Hh (sc-9024, Santa Cruz, reacts with all 3 Hh proteins) was incubated overnight at $4^{\circ} \mathrm{C}$. After 1-h incubation with universal

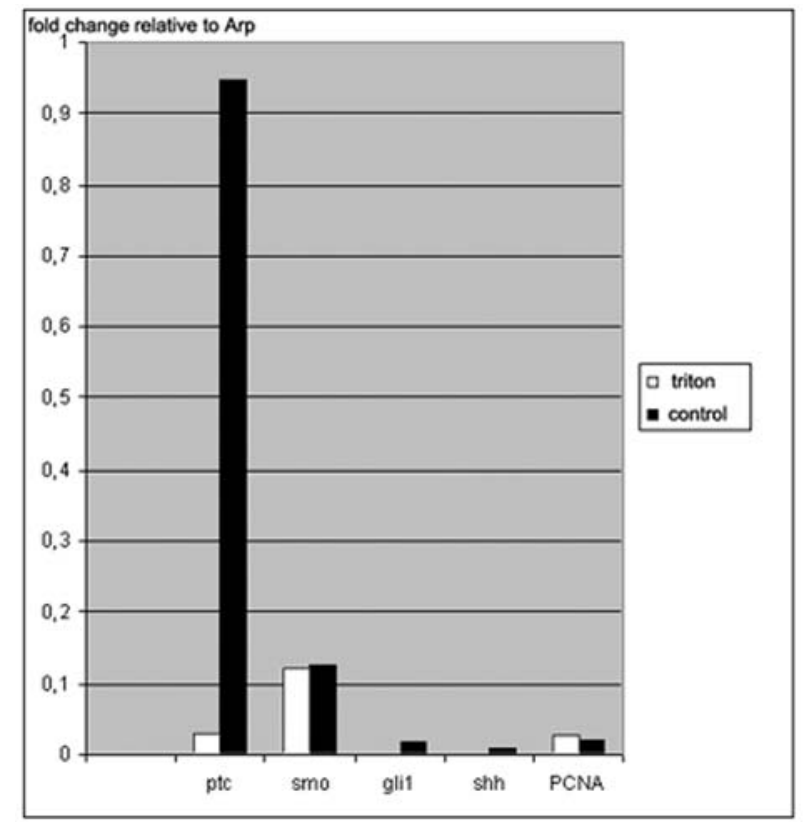

Figure 3. RT-PCR expression levels of PTCH1, SMO, SHH, GLI1 and PCNA (relative to ARP housekeeping gene) in PHD3 tumor (white columns) and in the control tissue (black columns).

Table III. Immunohistochemical analysis in early cancer stages.

\begin{tabular}{lll}
\hline $\begin{array}{l}\text { SCH tumor } \\
\text { tissue }\end{array}$ & Antibody & $\begin{array}{c}\text { Immunohistochemical } \\
\text { activity }\end{array}$ \\
\hline PHD1 & Ptch1 & Negative \\
& Gli1 & $\begin{array}{l}\text { Negative } \\
\text { Mildly positive }\end{array}$ \\
& Hh & Positive \\
PHD2 & Ptch1 & Negative \\
& Gli1 & Negative \\
\hline
\end{tabular}

secondary antibody (anti-goat, -mouse, -rabbit; Dako), the sections were incubated with PAP (rabbit, Dako) for $1 \mathrm{~h}$, then with DAB (Dako) for $7 \mathrm{~min}$, and counterstained with hematoxylin. As negative controls, the sections were processed in the same way but without the primary antibody.

\section{Results}

Typing with polymorphic markers. Typing with ten markers for the 9q22.3-31 region revealed a large deletion of about $10 \mathrm{Mb}$ in PHD3 tumor tissue (Table II), indicating loss of one entire allele in PTCH1 region. Haplotype analysis with the same markers (Fig. 2) was not particularly informative because the affected sister was not among the available relatives; the patient's $(\mathrm{SCH})$ haplotype for two markers (D9S176, D9S1783) is not replicated among the healthy family members. 

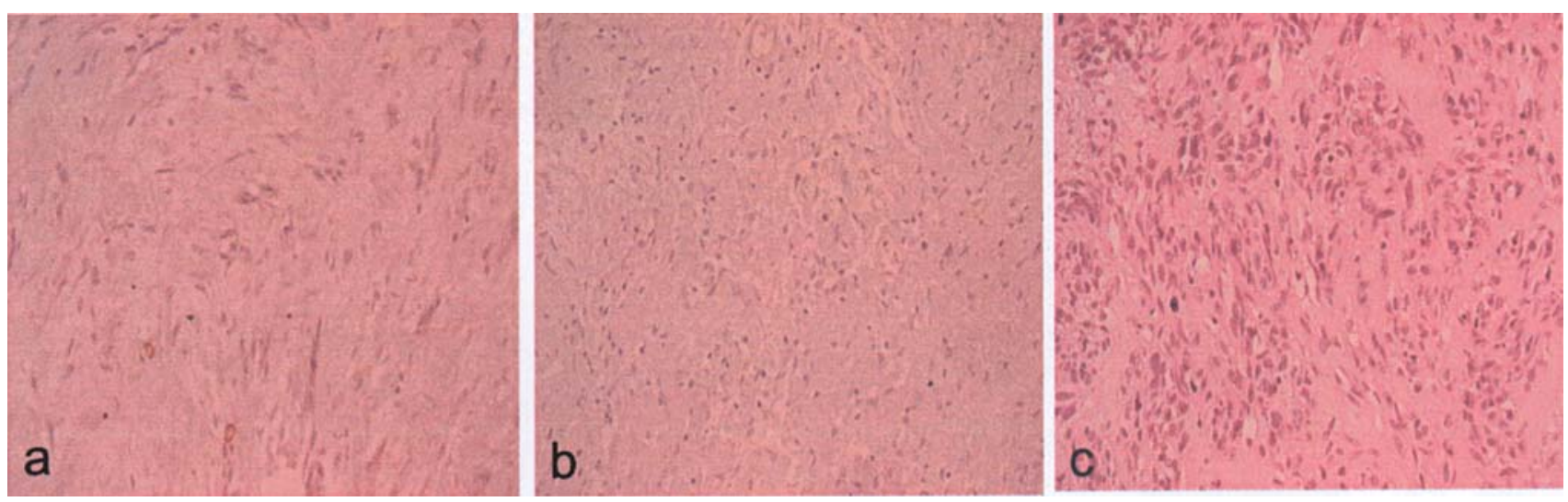

Figure 4. Immunohistochemical detection of Ptch1 (a), negative control (b), H\&E staining (c). Olympus camera 40-10; magnification x400.

None of three markers for the p16 region on 9p21 showed allelic loss (Table II); one marker was homozygous (D9S126). RFLP analysis of NF1 exon 5 did not show allelic loss (data not shown).

Mutation and methylation analysis. Neither dHPLC analysis nor subsequent sequencing of all PTCH1 exons revealed any mutations in the PHD3 tumor tissue (data not shown). Methylation analysis of PTCH1 promoter (20) did not show hypermethylation nor any mutations in the promoter sequence (data not shown). No mutations were detected in exon sequences of p16 gene (data not shown).

RT-PCR expression analysis. Expression levels of PTCH1, SHH and GLI1 in the PHD3 tumor tissue were by far lower than in the control sample. SMO was at the same level in both samples, while PCNA was slightly higher in the tumor (Fig. 3).

Protein expression in PHD1 and PHD2 tumors. Immunohistochemical analysis of Ptch1, Hh and Gli1 activity in early stages of malignancy showed significant increase of Ptch 1 protein level in the consecutive tumors (Table III and Fig. 4). PTCH1 seems to have been activated in PHD2 without detectable Hh signal (although possibly a mild signal had appeared in PHD1).

\section{Discussion}

MTT contains a substantial proportion of mature rhabdomyoblasts, but the tumor is derived from and behaves more like malignant schwannoma than rhabdomyosarcoma (4). Its molecular genetic basis is not known, which is not surprising because pathogenesis of schwannoma itself is not clear. Most schwannomas are associated with neurofibromatoses, and different genes are implicated in the syndrome sub-types: NF1 and NF2 genes were found altered in the respective tumors, and a new tumor suppressor gene is being investigated in schwannomatosis (which has recently been recognized as a nerofibromatosis distinct from the type 2). Malignant schwannomas appear to be mostly associated with NF1, but their low incidence in the syndrome and high proportion of sporadic cases do not suggest decisive role of NF1 gene in this malignancy.
In our family with two schwannoma cases, no association with neurofibromatoses was apparent, nor did RFLP analysis of NF1 gene in the MTT tissue indicate its involvement in the tumor. Regardless of the limited knowledge on schwannoma genesis, it can reasonably be supposed that rather complex genetic alterations are required for MTT development due to its extremely low incidence, and that some of these alterations are not typically involved in malignant schwannoma progression. For reasons outlined in the introduction, Hedgehog-Patched pathway malfunctioning seems to be a likely candidate for the MTT-specific alterations which convert malignant schwanoma into triton.

The large deletion we found on one Patched allele in PHD3 tumor clearly supports this hypothesis. Although mere haploinsufficiency of the PTCH1 gene, even at the constitutional level, causes usually mild dissorders known as Gorlin syndrome (NBCCS or BCNS), it has also been observed that the gene may retain one wild-type alelle in some tumors for which the syndrome predisposes. In particular, recent analysis of rhabdomyosarcoma on mouse models (9) showed that wild-type allele could be silenced by the promoter methylation, while the mutated PTC allele overexpressed non-functional protein.

However, we did not find increased PTCH1 promoter methylation in the PHD3 triton sample analysis (using the same procedure which gave positive results for some other neoplasia analyzed in our laboratory (20). Gli1 binding site was not methylated, and there were no mutations in the promoter sequence. This result does not help explain very low expression of PTCH1 observed in our PHD3 sample of MTT, which is so far below the control tissue level that it can hardly be attributed to haploinsufficiency alone. It is more likely that some PTCH1 silencing mechanism was also involved, and even non-homogeneous or reversible promoter methylation (undetectable in our sample) cannot be ruled out. The increased Patched expression in PHD2 sample, in comparison to PHD1, suggests the pathway activation at the onset of MTT transition from the former schwannoma. The PTCH1 autoregulatory response may have been inadequate due to the haploinsufficiency (although still visible during PHD2), but subsequent involvement of a silencing mechanism would better fit the expression profiles of PHD3.

Another explanation might be an alternative splicing of Ptch1 protein into a truncated form with a dominant negative 
phenotype. It has been shown that this isoform is normally present in brain and heart tissue, and it has been implicated in medulloblastoma formation. This truncated protein can abolish the activity of full-length protein, thus disrupting the pathway (21).

Apart from the PTCH1 deletion, no genetic changes were detected in the remaining assays. In particular, $\mathrm{LOH}$ analysis of the 9 p21 region and direct sequencing of p16 exons indicated no alterations in this gene that could contribute to the MTT proliferation. Also, haplotype investigation with PTCH1 region polymorphic markers, which was undertaken for four relatives of the affected family member, did not reveal anything outstanding about her haplotype.

Therefore, our results indicate only the involvement of Hedgehog-Patched pathway malfunctioning in MTT development. One Patched allele is deleted, the other is not mutated but its expression varies through different phases of tumor progression. In PHD3, expression of PTCH1, SHH and GLI1 is far lower than in the control neural tissue; only SMO, the gene that actually triggers the signal transduction to the nucleus, parallels the control level. It should be noted, though, that Hedgehog-Patched pathway malfunctioning is unlikely to be involved in the genesis of the primary cancer, i.e. the malignant schwannoma PHD1. In addition to previous arguments, restriction of the pathway role to MTT progression is also suported by the fact that malignant schwannomas have not been reported as a Gorlin syndrome feature (which is easily explained by very low incidence of the two conditions - provided that they do not share any underlying genetic causes).

Although a more reliable conclusion on the HedgehogPatched pathway involvement in MTT cannot be made before further cases are analyzed, the very possibility is worth considering due to the recent advances towards therapeutical exploitation of this pathway.

\section{References}

1. Nicolai P, Tomenzoli D, Berlucchi M, et al: Malignant Triton tumor of the ethmoid sinus and nasal cavity. Ann Otol Rhinol Laryngol 109: 880-886, 2000.

2. Mason P: Recklinghausen's neurofibromatosis, sensory neuromas and motor neuromas. In: Libman Anniversary. Vol. 2. International Press, New York, NY, pp793-802, 1932.

3. Woodruff JM and Perino G: Non-germ-cell or teratomatous malignant tumors showing additional rhabdomyoblastic differentiation, with emphasis on the malignant Triton tumor. Semin Diagn Pathol 11: 69-81, 1994.
4. Victoria L, McCulloch TM, Callaghan EJ, et al: Malignant Triton tumor of the head and neck: a case report and review of the literature. Head Neck 21: 663-670, 1999.

5. Mirsky R, Jessen KR, Brennan A, et al: Schwann cells as regulators of nerve development. J Physiol Paris 96: 17-24, 2002.

6. Woodruff JM, Kourea HP, Louis DN, et al: Schwannoma. In: Pathology and Genetics. Tumours of the Nervous System. WHO Classification of Tumours. Kleihues $\mathrm{P}$ and Cavenee WK (eds). IARC Press, Lyon, pp164-166, 2000.

7. Stecca B and Ruiz i Altaba A: Brain as a paradigm of organ growth: Hedgehog-Gli signaling in neural stem cells and brain tumors. J Neurobiol 64: 476-490, 2005.

8. Ruiz i Altaba A, Stecca B and Sanchez P: Hedgehog-Gli signaling in brain tumors: stem cells and paradevelopmental program in cancer. Cancer Lett 204: 145-157, 2004.

9. Uhmann A, Ferch U, Bauer R, et al: A model for PTCH1/Ptch1associated tumors comprising mutational inactivation and gene silencing. Int J Oncol 27: 1567-1575, 2005.

10. Atanasoki S, Boller D, De Ventura L, et al: Cell cycle inhibitors p21 and p16 are required for the regulation of Schwann cell proliferation. Glia 53: 147-157, 2005.

11. Gallo A, Suriano M, Simonelli M, et al: Recurrent malignant schwannoma of the parapharyngeal space in neurofibromatosis type 1. Ear Nose Throat J 82: 862-865, 2003.

12. Levanat S, Chidambaram A,Wicking C, et al: Pulsed-field gel electrophoresis and FISH mapping of chromosome 9q22: Placement of a novel zinc finger gene within the NBCCS and ESS1 region. Cytogenet Cell Genet 76: 208-213, 1997.

13. Louhelainen J, Lindstrom E, Hemminki K, et al: Dinucleotide repeat polymorphism within the tumor suppressor gene PTCH at 9q22. Clin Genet 54: 239-241, 1998.

14. Nagao K, Fujii K, Yamada M, et al: Identification of a novel polymorphism involving a CGG repeat in the PTCH gene and a genome-wide screening of CGG-containing genes. J Hum Genet 49: $97-101,2004$

15. Levanat S, Pavelic B, Crnic I, et al: Involvement of PTCH gene in various noninflammatory cysts. J Mol Med 78: 140-146, 2000.

16. Levanat S, Situm M, Crnic I, et al: Potential indicators of melanoma predisposition in relatives of familial cases. Alterations in CDK2A locus in cutaneous melanoma. Croat Med J 44: 418-424, 2003.

17. Levanat S, Gorlin RJ, Fallet S, et al: A two-hit model for developmental defects in Gorlin syndrome. Nat Genet 12: 8587, 1996.

18. Cacev T, Radosevic S, Spaventi R, et al: NF1 gene loss of heterozygosity and expression analysis in sporadic colon cancer. Gut 54: 1129-1135, 2005.

19. Regl G, Neill GW, Eichberger T, et al: Human GLI2 and GLI1 are part of a positive feedback mechanism in basal cell carcinoma. Oncogene 21: 5529-5539, 2002.

20. Cretnik M, Musani V, Oreskovic S, et al: Patched gene is epigenetically regulated in ovarian dermoids and fibromas, but not in BCCs. Int J Mol Med 19: 875-883, 2007.

21. Uchikawa H, Toyoda M, Nagao K, et al: Brain- and heartspecific Patched-1 containing exon $12 \mathrm{~b}$ is a dominant negative isoform and is expressed in medulloblastomas. Biochem Biophys Res Comm 349: 277-283, 2006. 